\title{
A Study on the Performance Analysis of a Batch Arrival Queue with Two Stages of Service, Bernoulli Schedule Vacation, Extended Vacation and Service Interruption
}

\author{
S. Maragathasundari \\ Asst Professor, Dept of Maths \\ Velammal Institute of Technology \\ Chennai, India
}

\author{
B. Balamurugan \\ Asso. Professor, Dept of Maths \\ Velammal Institute of Technology \\ Chennai, India
}

\begin{abstract}
In this paper a $M^{[\mathrm{X}]} / G / 1$ Queueing model with two stages of service is studied. Service interruption is considered as a major phenomenon. On completion of a service, the server will go for a vacation. An additional aspect of Optional extended vacation is considered in this model . In this model, repair process start immediately. Service time, Vacation time \& Repair time follows general distribution. Steady state solution \& Performance measures are derived.
\end{abstract}

\section{Mathematics Subject Classification: 60K25, $60 \mathrm{K30}$}

\section{Keywords}

Random breakdown, Repair process, extended vacation, Steady state, Queue size distribution.

\section{INTRODUCTION}

We study a single server batch arrival queue with poisson arrivals and general service times. The service is given in two stages. On completion of two stages of service, the server has the option of taking vacation. Here the vacation follows a Bernoulli schedule vacation. An additional phenomenon of extended vacation is considered here. During service, breakdown arrives at random. So repair process follows immediately.. Moreover the server has a option of taking a extended vacation with probability $r$ or continue service with probability 1-r. We assume that the service times, vacation times, breakdown times and extended vacation times each have a general distribution. Many researchers have made their efforts in queueing theory by considering various aspects like multi stages of service, Bernoulli vacation, Break down, repair process, general vacation and extended vacation. Madan [13, 14] studied a single server with two types of service with deterministic server vacation. Gautam Choudury et a;.[6] discussed a batch arrival with two phases of service with delay time in starting the repair process. Thangaraj and Vanitha [15] have obtained transient solution of two stage heterogeneous service with compulsory vacation and random breakdowns .Madan[2] discussed a queueing model with random failures and delayed repairs. Takagi[4] investigated time dependent analysis of $\mathrm{M} / \mathrm{G} / 1$ vacation models with exhaustive service. This paper is organized as follows. The model assumption is given in section 2 . In section 3 all the equations governing the mathematical system in the steady state are formulated. The supplementary variable technique is used in this section to obtain the closed form of the probability generating function of the queue length, average queue size and the average waiting time are given in section 4

\section{MODEL ASSUMPTIONS}

a) Customers arrive at the system in batches of variable size in a compound Poisson process and they are provided one by one service on a 'first come'-first served basis. Let $\lambda c_{i}(i=$

$1,2,3 \ldots$ ) be the first order probability that a batch of $i$ customers arrives at the system during a short interval of time $(t, t+d t)$, where $0 \leq c_{i} \leq 1$ and $\sum_{i=1}^{\infty} c_{i}=1$ and $\lambda>0$ is the mean arrival rate of batches

b) Each customer undergoes two stages of service provided by a single server on a first come first served basis. The service time follows general(arbitrary)distribution with distribution function $\mathrm{G}(s)$ and density function $\mathrm{g}(s)$. Let $\mu(x) d x$ be the conditional probability density of service completion during the interval $(x, x+d x)$,given that the elapsed time is $x$,so that

$$
\mu(x)=\frac{g(x)}{1-G(x)}
$$

and therefore

$$
g(s)=\mu(s) e^{-\int_{0}^{s} \mu(x) d x} \quad i
$$$$
=1,2
$$

c) As soon as a service is completed, the server may go for a vacation. Also the server may go for a extended vacation with probability $\mathrm{r}$ or return back to the system to continue the service for the next customer with probability 1-r. Let $\beta(x) d x$ be the conditional probability of a completion of a vacation during the interval $(x, x+d x)$,so that

d)The server's vacation time follows a general(arbitrary)

$$
\beta(x)=\frac{m(x)}{1-M(x)}
$$

And, therefore

$m(s)=\beta(s) e^{-\int_{0}^{s} \beta(x) d x}$ with distribution function $M(s)$ and density function $m(s)$.

The server's Extended vacation time follows a general(arbitrary) distribution with distribution function $H(s)$ and density function $h(s)$. Let $\theta(x) d x$ be the conditional probability of a completion of a Extended vacation during the interval $(x, x+d x)$, so that

$$
\theta(x)=\frac{h(x)}{1-H(x)}
$$

And, therefore

$$
h(s)=\theta(s) e^{-\int_{0}^{s} \theta(x) d x}
$$


e) The system may breakdown at random and breakdowns are assumed to occur according to a Poisson stream with mean breakdown rate $\alpha>0$.)

f)The server's repair time follows a general(arbitrary) distribution with distribution function $F(x)$ and density function $f(x)$. Let $\gamma(x) d x$ be the conditional probability of a completion of a repair during the interval $(x, x+d x)$, so that

Repair time $\gamma(x)=\frac{f(x)}{1-F(x)} \quad$ and $f(s)=\gamma(s) e^{-\int_{0}^{s} \gamma(x) d x}$ (5)

g) Various stochastic process involved in the system are assumed to be independent of each other.

\section{EQUATIONS GOVERNING THE SYSTEM}

The equations governing the system are as follows:

$\frac{\partial}{\partial x} P_{n}^{(1)}(x, t)+\frac{\partial}{\partial x} P_{n}^{(1)}(x, t)+\frac{\partial}{\partial x}\left(\lambda+\mu_{1}(x)+\alpha\right) P_{n}^{(1)}(x, t)=$ $\lambda \sum_{i=1}^{n-1} C_{i} P_{n-i}^{(1)}(x, t) n \geq 1$

(6)

$\underset{0}{\frac{\partial}{\partial x}} P_{0}^{(1)}(x, t)+\frac{\partial}{\partial x} P_{0}^{(1)}(x, t)+\frac{\partial}{\partial x}\left(\lambda+\mu_{1}(x)+\alpha\right) P_{0}^{(1)}(x, t)=$

$\frac{\partial}{\partial x} P_{n}^{(2)}(x, t)+\frac{\partial}{\partial x} P_{n}^{(1)}(x, t)+\frac{\partial}{\partial x}\left(\lambda+\mu_{2}(x)+\alpha\right) P_{n}^{(2)}(x, t)=$ $\lambda \sum_{i=1}^{n-1} C_{i} P_{n-i}^{(2)}(x, t)$

(8)

$\frac{\partial}{\partial x} P_{0}^{(2)}(x, t)+\frac{\partial}{\partial x} P_{0}^{(2)}(x, t)+\frac{\partial}{\partial x}\left(\lambda+\mu_{2}(x)+\alpha\right) P_{0}^{(2)}(x, t)=$

$\frac{\partial}{\partial x} V_{n}(x, t)+\frac{\partial}{\partial x} V_{n}(x, t)+\frac{\partial}{\partial x}(\lambda+\beta(x)+\delta) V_{n}(x, t)=$ $\lambda \sum_{i=1}^{n-1} C_{i} V_{n-i}(x, t)+\delta V_{n+1}(x) \quad n \geq 1$

(10)

$\frac{\partial}{\partial x} V_{o}(x, t)+\frac{\partial}{\partial x} V_{o}(x, t)+(\lambda+\beta(x)) V_{o}(x, t)=\delta V_{1}(x)$

(11)

$\frac{\partial}{\partial x} E_{n}(x, t)+\frac{\partial}{\partial x} E_{n}(x, t)+\frac{\partial}{\partial x}(\lambda+\theta(x)) E_{n}(x, t)=$ $\lambda \sum_{i=1}^{n-1} C_{i} E_{n-i}(x, t) \quad n \geq 1$

$\frac{\partial}{\partial x} E_{0}(x, t)+\frac{\partial}{\partial x} E_{0}(x, t)+\frac{\partial}{\partial x}(\lambda+\theta(x)) E_{0}(x, t)=0$

(13)

$\frac{\partial}{\partial x} R_{n}(x, t)+\frac{\partial}{\partial x} R_{n}(x, t)+(\lambda+\gamma(x)) R_{n}(x, t)=$ $\lambda \sum_{i=1}^{n-1} C_{i} R_{n-i}(x, t)$

(14)

$\frac{\partial}{\partial x} R_{0}(x, t)+\frac{\partial}{\partial x} R_{0}(x, t)+(\lambda+\gamma x) R_{0}(x, t)=0$

(15)

As $t \rightarrow \infty$, the above equation becomes

$\lambda Q=\int_{0}^{\infty} R_{0}(x) \gamma(x) d x+(1-p) \int_{0}^{\infty} P_{0}^{(2)}(x) \mu_{2}(x) d x$ $+(1-r) \int_{0}^{\infty} V_{0}(x) \beta(x) d x+\int_{0}^{\infty} E_{0}(x) \theta(x) d x$

(16)

The following boundary Conditions are used to solve the above equations:

$P_{n}^{(1)}(0)=$

$(1-p) \int_{0}^{\infty} P_{n+1}^{(2)}(x) \mu_{2}(x) d x+(1-r) \int_{0}^{\infty} V_{n+1}(x) \beta(x) d x$

$+\int_{0}^{\infty} E_{n+1}(x) \theta(x) d x \int_{0}^{\infty} R_{n+1}(x) \gamma(x) d x+\lambda C_{n+1} Q$

(17)

$P_{n}^{(2)}(0)=\int_{0}^{\infty} P_{n}^{(1)}(x) \mu_{1}(x) d x$

(18)

$V_{n}(0)=p \int_{0}^{\infty} P_{n}^{(2)}(x) \mu_{2}(x) d x$

(19)

$E_{n}(0)=r \int_{0}^{\infty} V_{n}(x) \beta(x) d x$

$R_{n}(0)=\alpha\left[\int_{0}^{\infty} P_{n}^{(1)}(x) d x+\int_{0}^{\infty} P_{n}^{(2)}(x) d x\right]$

$R_{n}^{(1)}(0)=\int_{0}^{\infty} D_{n}(x) \delta(x) d x$

(22)

$R_{0}(0)=0$

(23)

Multiplying Equation (6) by $Z^{n}$ sum over $n$ from 1 to $\infty$ and adding to (7), using Probability generating function, we obtain $\frac{\partial}{\partial x} P_{q}^{(1)}(x, z)+\left(\lambda-\lambda(z)+\mu_{1}(x)+\alpha\right) P_{q}^{(2)}(x, z)=0$ (24)

$\frac{\partial}{\partial x} P_{q}^{(2)}(x, z)+\left(\lambda-\lambda(z)+\mu_{2}(x)+\alpha\right) P_{q}^{(2)}(x, z)=0$

(25)

$\frac{\partial}{\partial x} V_{q}(x, z)+(\lambda-\lambda(z)+\beta(x)) V_{q}(x, z)=0$

(26)

$\frac{\partial}{\partial x} R_{q}(x, z)+(\lambda-\lambda(z)+\gamma(x)) R_{q}(x, z)=0$

(27)

$\frac{\partial}{\partial x} E_{q}(x, z)+(\lambda-\lambda(z)+\theta(x)) E_{q}(x, z)=0$

(28)

Multiplying Equation (17) by $z^{n+1}$, sum over $n$ from 0 to $\infty$, $z P_{q}^{(1)}(0, z)=(1-p) \int_{0}^{\infty} P_{q}^{(2)}(x, z) \mu_{2}(x) d x+(1-$

r) $\int_{0}^{\infty} V_{q}(x, z) \beta(x) d x+$

$\int_{0}^{\infty} R_{q}(x, z) \gamma(x) d x+\int_{0}^{\infty} E_{q}(x, z) \theta(x) d x-\lambda Q(C(z)-1)$ (29)

$P_{q}^{(2)}(0, z)=\int_{0}^{\infty} P_{q}^{(1)}(x, z) \mu_{1}(x) d x$

(30)

$P_{q}^{(3)}(0, z)=\int_{0}^{\infty} P_{q}^{(2)}(x, z) \mu_{2}(x) d x$

(30a)

$V_{q}(0, z)=p \int_{0}^{\infty} P_{q}^{(2)}(x, z) \mu_{2}(x) d x$

(31)

$E_{q}(0, z)=r \int_{0}^{\infty} V_{q}(x, z) \beta(x) d x$

(32) 
$R_{q}(0, z)=\alpha z\left[\int_{0}^{\infty} P_{q}^{(1)}(x, z) d x+\int_{0}^{\infty} P_{q}^{(2)}(x, z) d x\right]=$ $\alpha z\left[P_{q}^{(1)}(z)+P_{q}^{(2)}(z)\right]$

Integrating Equation (24) from 0 to $\infty$

$P_{q}^{(1)}(x, z)=P_{q}^{(1)}(0, z) e^{-(\lambda-\lambda C(z)+\alpha) x-\int_{0}^{\infty} \mu_{1}(t) d t}$

(34)

Let $\lambda-\lambda C(z)+\alpha=m$

Again Integrating the above equation, by parts with respect to $x$,

$P_{q}^{(1)}(z)=P_{q}^{(1)}(0, z)\left(\frac{1-\overline{G_{1}}(m)}{m}\right)$

(35)

Where $\overline{G_{1}}(m)=\int_{0}^{\infty} e^{-(\lambda-\lambda C(z)+\alpha) x} d G_{1}(x)$ is Laplace

Stieltjes Transform of the service time $G_{1}(x)$.

Multiply both sides of Equation (34) by $\mu(x) \&$ integrating over $x$, we get

$\int_{0}^{\infty} P_{q}^{(1)}(x, z) \mu_{1}(x) d x=P_{q}^{(1)}(0, z) \overline{G_{1}}(m)$

(36)

Similarly from equations (26), (27),(28) \& (29) applying the same process we have,

$P_{q}^{(2)}(z)=\frac{P_{q}^{(2)}(0, z)\left(1-\overline{G_{2}}(m)\right)}{m}$

(36a)

$$
\begin{aligned}
\int_{0}^{\infty} P_{q}^{(2)}(x, z) \mu_{2}(x) d x & =P_{q}^{(2)}(0, z) \overline{G_{2}}(m) \\
& =\left[\int_{0}^{\infty} P_{q}^{(1)}(x, z) \mu_{1}(x) d x\right] \overline{G_{2}}(m) \\
& =P_{q}^{(1)}(0, z) \overline{G_{1}}(m) \overline{G_{2}}(m)
\end{aligned}
$$

From (27), using (32) \& (33), we have

$V_{q}(o, z)=p P_{q}^{(1)}(0, z) \overline{G_{1}}(m) \overline{G_{2}}(m)$

(38)

Integrating equation (22) from 0 to $x$, we have

$V_{q}(x, z)=V_{q}(o, z) e^{-(\lambda-\lambda C(z)+\alpha) x-\int_{0}^{x} \beta(t) d t}$

$p P_{q}^{(1)}(0, z) \overline{G_{1}}(m) \overline{G_{2}}(m) e^{-\left(\lambda-\lambda C(z)+\alpha+\delta-\frac{\delta}{z}\right) x-\int_{0}^{x} \beta(t) d t}$ (39)

Let $s=\lambda-\lambda C(z)+\delta-\frac{\delta}{z}$

Integrating equation (35) by parts with respect to $x$, we have

$V_{q}(z)=\frac{p P_{q}^{(1)}(0, z) \overline{G_{1}}(m) \overline{G_{2}}(m)(1-\bar{K}(s))}{s}$

(40)

Where $\bar{K}(s)=\int_{0}^{\infty} e^{-\left(\lambda-\lambda C(z)+\alpha+\delta-\frac{\delta}{z}\right) x} d K(x)$ is the Laplace Stieltjes transform of the vacation time $K(x)$.
Multiplying equation (35) both $\beta(x) \&$ integrating over $x$, we get

$\int_{0}^{\infty} V_{q}(x, z) \beta(x) d x=p P_{q}^{(1)}(0, z) \overline{G_{1}}(m) \overline{G_{2}}(m) \bar{K}(s)$

Applying the same process for equation (23) \& (24)

$E_{q}(z)=\frac{r p P_{q}^{(1)}(0, z) \overline{G_{1}}(m) \overline{G_{2}}(m) \bar{K}(s)(1-\bar{H}(w))}{w}$

And therefore

$\left[\begin{array}{c}\int_{0}^{\infty} E_{q}(x, z) \theta(x) d x=r p P_{q}^{(1)}(0, z) \overline{G_{1}}(m) \overline{G_{2}}(m) \bar{K}(s) \bar{H}(w) \\ \text { Where } \bar{H}(w)=\int_{0}^{\infty} e^{-(\lambda-\lambda C(z)) x} d H(x) \text { is the Laplace } \\ \text { Stieltjes transform of the Extended vacation } H(x)\end{array}\right]$

Also we have, integrating (24) from 0 to $x$

$R_{q}(x, z)=R_{q}(0, z)\left[e^{-\left(\lambda-\lambda C(z)+\delta-\frac{\delta}{z}\right) x-\int_{0}^{x} \gamma(t) d t}\right]$

$\alpha z P_{q}^{(1)}(0, z)\left[\frac{1-\overline{G_{1}}(m)}{m}+\right.$

$\left.\frac{\overline{G_{1}}(m)\left(1-\overline{G_{2}}(m)\right)}{m}\right]\left[e^{-\left(\lambda-\lambda C(z)+\delta-\frac{\delta}{z}\right) x-\int_{0}^{x} \gamma(t) d t}\right]$

(44)

Integrating (40) by parts with respect to $x$, we obtain

$R_{q}(z)=\frac{\alpha z P_{q}^{(1)}(0, z)\left(1-\overline{G_{1}}(m) \overline{G_{2}}(m)\right)(1-\bar{F}(s))}{m s}$

Multiply Equation (40) by $\gamma(x)$ on both sides and integrating with respect to $x$, we get

$\int_{0}^{\infty} R_{q}(x, z) \gamma(x) d x=\frac{\alpha z P_{q}^{(1)}(0, z)\left(1-\overline{G_{1}}(m) \overline{G_{2}}(m) \bar{F}(s)\right)}{m s}$ (46)

Using Equation (33),(37), (39) and (42) Equation (25) becomes,

$$
\begin{array}{r}
z P_{q}^{(1)}(0, z)=(1-p) P_{q}^{(1)}(0, z) \overline{G_{1}}(m) \overline{G_{2}}(m) \\
\quad+p P_{q}^{(1)}(0, z) \overline{G_{1}}(m) \overline{G_{2}}(m) \bar{K}(s) \\
+r p P_{q}^{(1)}(0, z) \overline{G_{1}}(m) \overline{G_{2}}(m) \bar{K}(s) \bar{H}(w) \\
+\frac{\alpha z P_{q}^{(1)}(0, z)\left(1-\overline{G_{1}}(m) \overline{G_{2}}(m) \bar{F}(s)\right)}{m} \\
+\lambda Q(C(z)-1)
\end{array}
$$

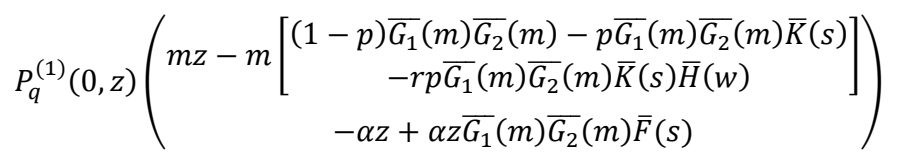$$
=m \lambda Q(C(z)-1)
$$

$P_{q}^{(1)}(0, z)=\frac{m \lambda Q(C(z)-1)}{D r}=\frac{N r}{D r}$

$N r=m \lambda Q(C(z)-1)=\bar{m} Q w$

$\mathrm{Dr}=$

$m\left(z-\overline{G_{1}}(m) \overline{G_{2}}(m)(1-p-p \bar{K}(s)-r p \bar{K}(s) \bar{H}(w))\right)-$ $\alpha z+\alpha z \overline{G_{1}}(m) \overline{G_{2}}(m) \bar{F}(s)(49)$ 
Therefore

$$
P_{q}^{(1)}(z)=\frac{\bar{m} Q w}{m\left(z-\overline{G_{1}}(m) \overline{G_{2}}(m)(1-p-p \bar{K}(s)-r p \bar{K}(s) \bar{H}(w))\right)} \frac{1-\overline{G_{1}}(m)}{m}
$$

$$
\begin{aligned}
& P_{q}^{(2)}(z)= \\
& \frac{\bar{m} Q w}{m\left(z-\overline{G_{1}}(m) \overline{G_{2}}(m)(1-p-p \bar{K}(s)-r p \bar{K}(s) \bar{H}(w))\right)} \frac{1-\overline{G_{1}}(m) \overline{G_{2}}(m)}{m} \\
& -\alpha z+\alpha z \overline{G_{1}}(m) \overline{G_{2}}(m) \bar{F}(s)
\end{aligned}
$$

$$
\begin{aligned}
& V_{q}(z)= \\
& \begin{array}{l}
m\left(z-\overline{G_{1}}(m) \overline{G_{2}}(m)(1-p-p \bar{K}(s)-r p \bar{K}(s) \bar{H}(w))\right) \\
-\alpha z+\alpha z \overline{G_{1}}(m) \overline{G_{2}}(m) \bar{F}(s)
\end{array}
\end{aligned}
$$

$$
E_{q}(z)=
$$

$\frac{\bar{m} Q w}{m\left(z-\overline{G_{1}}(m) \overline{G_{2}}(m)(1-p-p \bar{K}(s)-r p \bar{K}(s) \bar{H}(w))\right)} \frac{r p \overline{G_{1}}(m) \overline{G_{2}}(m) \bar{K}(s)(1-\bar{H}(w))}{w}$

$R_{q}(z)=$

$\frac{\bar{m} Q w}{m\left(z-\overline{G_{1}}(m) \overline{G_{2}}(m)(1-p-p \bar{K}(s)-r p \bar{K}(s) \bar{H}(w))\right)} \frac{\alpha z\left(1-\overline{G_{1}}(m) \overline{G_{2}}(m)\right)(1-\bar{F}(s))}{m s}$
(54)

Let $M_{q}(z)$ denotes the probability generating function of the queue size

$M_{q}(z)=P_{q}^{(1)}(z)+P_{q}^{(2)}(z)+V_{q}(z)+E_{q}(z)+R_{q}(z)$

(55)

In order to obtain $Q$, We use the normalization condition

$M_{q}(1)+Q=1$

(56)

\section{THE AVERAGE QUEUE SIZE AND THE AVERAGE WAITING TIME}

Let $L_{q}$ denote the mean number of customers in the queue under the steady state. Then

$L_{q}=\left.\frac{d}{d z} M_{q}(z)\right|_{z=1}$

Since $S_{q}(z)=0 / 0$ at $z=1$, we use the result

$$
\begin{aligned}
& L_{q}=\lim _{z \rightarrow 1} \frac{d}{d z} M_{q}(z) \\
& =P_{q}(1)=\lim _{z \rightarrow 1} \frac{D^{\prime}(z) N^{\prime \prime}(z)-N^{\prime}(z) D^{\prime \prime}(z)}{2\left(D^{\prime}(z)\right)^{2}} \\
& =\frac{D^{\prime}(1) N^{\prime \prime}(1)-N^{\prime}(1) D^{\prime \prime}(1)}{2\left(D^{\prime}(1)\right)^{2}}
\end{aligned}
$$

$N^{\prime}(1)=\lambda Q E(I)\left[1-\overline{G_{1}}(\alpha)+\overline{G_{1}}(\alpha) \overline{G_{2}}(\alpha)\right]$

(57)

$N^{\prime \prime}(1)=$

$2 \lambda Q E(I)\left[\lambda E(I) \overline{G^{\prime}}{ }_{1}(\alpha)+(-\lambda E(I))\left[\overline{G^{\prime}}{ }_{1}(\alpha) \overline{G_{2}}(\alpha)+\right.\right.$
$\left.\overline{G_{1}}(\alpha) \overline{G_{2}^{\prime}}(\alpha)\right]-p \alpha \overline{G_{1}}(\alpha) \overline{G_{2}}(\alpha) E(K)+$ $\left.r p \alpha \overline{G_{1}}(\alpha) \overline{G_{2}}(\alpha) E(K)+\alpha\left(1-\overline{G_{1}}(\alpha) \overline{G_{2}}(\alpha)\right)(-E(F))\right]+$ $\lambda Q E\left(I(I-1)\left[1-\overline{G_{1}}(\alpha)+\overline{G_{1}}(\alpha) \overline{G_{2}}(\alpha)\right]\right.$

(58)

$D^{\prime}(1)=-\lambda E(I)\left[1-\overline{G_{1}}(\alpha) \overline{G_{2}}(\alpha)(1-p-p-r p)\right]+$ $\alpha\left[1-\left\{\left(-\lambda E(I)\left(\overline{G_{1}^{\prime}}(\alpha) \overline{G_{2}}(\alpha)+\overline{G_{1}}(\alpha) \overline{G^{\prime}}{ }_{2}(\alpha)\right)\right)(1-p-\right.\right.$ $p-r p)+\overline{G_{1}}(\alpha) \overline{G_{2}}(\alpha)(-p E(K)(-\lambda E(I)+\delta))-$ $r p[E(k)(-\lambda E(I)+\delta)+E(H)(-\lambda E(I))]\}]$ (59)

$D^{\prime \prime}(1)=-\lambda E(I(I-1))\left[1-\overline{G_{1}}(\alpha) \overline{G_{2}}(\alpha)(1-2 p-r p)\right]+$ $2(-\lambda E(I))[1-$

$\left\{-\lambda E(I)\left(\overline{G_{1}^{\prime}}(\alpha) \overline{G_{2}}(\alpha)+\overline{G_{1}}(\alpha) \overline{G_{2}^{\prime}}(\alpha)\right)(1-2 p-r p)+\right.$

$\overline{G_{1}}(\alpha) \overline{G_{2}}(\alpha)(-p E(k)(-\lambda E(I)+\delta))-r p[E(K)(-\lambda E(I)+$ $\delta)+E(H)(-\lambda E(I))]\}]+$

$\alpha\left\{\left[-\lambda E(I(I-1))\left(\overline{\bar{G}_{1}^{\prime}}(\alpha) \overline{G_{2}}(\alpha)+\overline{G_{1}}(\alpha) \overline{G^{\prime}}{ }_{2}(\alpha)\right)\right](1-\right.$ $2 p-r p)+(\lambda E(I))^{2}\left[\overline{G^{\prime \prime}}{ }_{1}(\alpha) \overline{G_{2}}(\alpha)+2 \overline{G_{1}^{\prime}}(\alpha) \overline{G_{2}^{\prime}}(\alpha)+\right.$ $\left.\overline{G_{1}}(\alpha) \overline{G^{\prime \prime}}{ }_{2}(\alpha)\right](1-2 p-r p)-\lambda E(I)\left[\overline{G^{\prime}}{ }_{1}(\alpha) \overline{G_{2}}(\alpha)+\right.$ $\left.\overline{G_{1}}(\alpha) \overline{G_{2}^{\prime}}(\alpha)\right][-(-\lambda E(I) E(H))]+$ $(-\lambda E(I))\left[\left(\overline{G_{1}^{\prime}}(\alpha) \overline{G_{2}}(\alpha)+\right.\right.$ $\left.\left.\overline{G_{1}}(\alpha) \overline{G_{2}^{\prime}}(\alpha)\right)\right][-p E(K)(-\lambda E(I)+\delta) E(K)]-$ $r p[(-\lambda E(I)+\delta) E(K)-\lambda E(I) E(H)]+$ $(-\lambda E(I))\left(\overline{G_{1}^{\prime}}(\alpha) \overline{G_{2}}(\alpha)+\right.$ $\left.\overline{G_{1}}(\alpha) \overline{G^{\prime}}{ }_{2}(\alpha)\right)[-p E(K)(-\lambda E(I)+\delta)-r p[(-\lambda E(I)+$ $\delta) E(K)+(-\lambda E(I) E(H))]]+\overline{G_{1}}(\alpha) \overline{G_{2}}(\alpha)(-p[(-\lambda E(I)+$ $\left.\left.\delta)^{2} E\left(K^{2}\right)+E(K)(-\lambda E(I)(I-1)-2 \delta)\right]\right)-$ $r p\left\{\left((-\lambda E(I)+\delta)^{2} E\left(K^{2}\right)+E(K)(-\lambda E(I(I-1)-2 \delta)+\right.\right.$ $E(K)(-\lambda E(I)+\delta)(-\lambda E(I) E(H))+(-\lambda E(I(I-$ 1) $E(H))+(-\lambda E(I))^{2} E\left(H^{2}\right)-\lambda E(I) E(H)(-\lambda E(I)+$ $\delta) E(K))\}\}$

Where primes and double primes in (56) denote first and second derivatives at $z=1$, respectively.Carrying out the derivatives at $z=1$ and if we substitute the values of $N^{\prime}(1), N^{\prime \prime}(1), D^{\prime}(1)$ and $D^{\prime \prime}$ (1) into (56) we obtain $L_{q}$ in closed form. Further, the mean waiting time of a customer could be found using $W_{q}=L_{q} / \lambda$.

\section{CONCLUSIONS}

In this paper, we study a batch arrival queue with two stages of service, vacation, extended vacation and service interruptions. The average number of customers in the queue is found using the Supplementary variable method. This paper clearly analyses the steady state results and some performance measures of the model. As a future work in this model, various aspects like multi stages of service, optional repair, deterministic service time and delay time can be discussed.

\section{REFERENCES}

[1] Chodhury. G and Madan. K.C (2005), "A two stage batch arrival queueing systems with a modified Bernoulli schedule vacation under N-policy", Mathematical and computer Modelling, Vol.42, pp.71-85.

[2] K.C Madan (1994), "A queueing system with random failures and delayed repairs", J. Ind Statist. Assoc, Vol.32, pp.39-48. 
[3] B.T Doshi (1986), "Queueing systems with Vacation - a Survey”, Queueing Systems, Vol.1, pp.29-66.

[4] Takagi. H (1990), "Time-dependent analysis of M/G/1 vacation with exhaustive service", Queueing Systems, Vol.6, pp.369-390.

[5] Chodhury. G and Madan. K.C (2004), "A two phase batch arrival queueing system with a vacation time under Bernoulli schedule", Applied mathematics and Computation, Vol. 149, pp.337-349.

[6] G. Choudhury, L. Tadj and M. Paul(2007), "Steady state analysis of an $\mathrm{M}^{[\mathrm{x}]} / \mathrm{G} / 1$ queue with two phase service and Bernoulli vacation schedule under multiple vacation policy ", Applied Mathematical Modeling Vol.31, No.3, pp.1079-1091.

[7] Maraghi. F.A, Madan . K.C and Darby-Dowman. K (2009), "Batch Arrival queueing system with Random Breakdowns and Bernoulli Schedule server vacations having General vacation Time Distribution", International Journal of Information and Management Sciences, Vol.20, pp.55-70.

[8] Takine . T (2001), "Distributional form of Little's law for FIFO queues with multiple Markovian arrival streams and its application to queues with vacations", Queueing Systems, Vol.37, pp.31-63.

[9] Chodhury. G (2002), "Some aspects of M/G/1 queue with two different times under multiple vacation policy", Stochasic Analysis and applications, Vol.20, No.5, pp.901-909.

[10] Madan .K.C and Abu-Dayyeh .W. \& Saleh (2002), “ An M/G/1 queue with second optional service and Bernoulli schedule server vacations", Systems Science, Vol.28, No.3, pp.51-62.
[11] Madan . K.C, Al-Rawi, Z.R \& Al-Nasser, A.D (2005), "On $\mathrm{Mx} /(\mathrm{G} 1 \mathrm{G} 2) / 1 / \mathrm{G}(\mathrm{BS}) / \mathrm{Vs}$ vacation queue with two types of general heterogeneous service", Journal of Applied mathematics and Decision sciences, Vol.3, pp.123-135.

[12] Madan .K.C and Chodhury. G (2006), “ Steady state analysis of an $M^{[x]} /(G 1 G 2) / 1$ queue with restricted admissibility and random set up time, International Journal of Information and Management sciences",Vol.17, No.2,pp.33-56.

[13] K.C Madan.(2001),“On a single server queue with two stage heterogeneous service and deterministic server vacations", International journal of system sciences, Vol.32, pp.837-844.

[14] K.C Madan and R.F Anabasi (2001), "A single server queue with two types of service, Bernoulli schedule server vacations and a single vacation policy", Pakistan journal of statistics, Vol.19, pp331-342.

[15] V. Thangaraj and Vanitha (2010), $\mathrm{M}^{\mathrm{l}} / \mathrm{G} / 1$ queue with Two Stage Heterogeneous service Compulsory server vacation and Random breakdowns", Int Journal of Contemp .Math. Sciences”, Vol.5, pp.307-322.

[16] Maragathasundari. S and Srinivasan. S (2014a), "A NonMarkovian Multistage Batch arrival queue with breakdown and reneging", Mathematical problems in engineering, Volume 2014/16 pages/ Article ID .519579/ http: // dx. doi. Org / 10.1155/2014/ 519579.

[17] Maragathasundari. S and Srinivasan. S (2014b), "Analysis of Batch arrival queue with two stages of service and phase vacations", Missouri Journal of Mathematical Sciences, Vol. FALL 2014, No.2, pp.189205. 